\title{
Error-Convergence Property of ab-initio Finite-Element Calculation with Curving Grid*
}

\author{
Yoshinori SHIIHARA ${ }^{* *}$, Osamu KUWAZURU** and Nobuhiro YOSHIKAWA ** \\ **Institute of Industrial Science, The University of Tokyo, \\ 4-6-1 Komaba, Meguro-ku, Tokyo, Japan \\ E-mail: nori@telu.iis.u-tokyo.ac.jp
}

\begin{abstract}
In order to estimate the material property with sufficient accuracy and acceptable computational time using ab-initio calculation, the reduction of degrees of freedom required to approximate highly oscillated wave function around the nuclei is necessary. The curving-grid mesh which allocates a fine mesh around the nuclei seems effective for the reduction. We investigate the convergence property for $a b$-initio finite-element calculation with curving-grid mesh in terms of total energy. Through the calculation of an oxygen molecule, we found that the favorable convergence property, i.e. the variational convergence with the uniform rate, of the finite element method with uniform mesh is conserved. The application to bulk rock-salt magnesium oxide including 216 atoms shows the effectiveness of the curving-grid mesh in large-scale ab-initio calculation.
\end{abstract}

Key words: Quantum Mechanics, Density Functional Theory, Finite Element Method, Mesh Refinement Technique, Error Analysis

\section{Introduction}

Nowadays ab-initio calculation based on the Density Functional Theory (DFT) ${ }^{(1)}$ has been regarded as an indispensable tool to design the functional materials applied to the electronic devices ${ }^{(2)}$, catalysts ${ }^{(3)}$, fuel cells ${ }^{(4)}$ and so on. From the total energy or its derivative obtained by ab-initio calculation, one can predict various material properties with reliable accuracy such as ideal elastic properties, elastic constants, bulk modulus, shear modulus, ideal strength ${ }^{(5)-(8)}$ and so on, even in unknown combinations of materials. In the ab-initio calculation in a large-scale and complex system such as ceramics/metal interface, large number of degrees of freedom are required to calculate the total energy with high accuracy, since fine resolution is required to represent the variation of wave function highly oscillated around the nuclei. Thus we should circumvent this problem to calculate reliable values of material property with acceptable computational time. Concerning the plane wave $\operatorname{method}^{(9)}$, for the most progressive ab-initio calculation methodology in condensed matter physics, the norm-conserving pseudopotential method ${ }^{(10)}$ is developed as a very effective way to reduce the degrees of freedom. We only treat the valence wave function in the calculation, since effects of core electrons to valence electrons are put into the pseudopotential. Moreover, the number of degrees of freedom necessary to represent the wave function is significantly reduced because the wave functions become much smoother than the all-electron wave functions due to the nodeless property of the pseudo-wave function in the core region. Using the curving-grid mesh is another idea for the reduction. Even in the pseudopotential techniques, the wave function is highly localized near the nuclei, especially in first-row atoms or transition atoms. The deformation-function method $^{(11)}$ transforms the uniform Euclidean coordinate to the curviliner coordinate and 
generates the curving-grid mesh, in which grid points are densely allocated around the nuclei. The effectiveness of this method in the plane wave method has been proved through the calculation for bulk ceramics; $\mathrm{TiO}_{2}{ }^{(12)}$ and $\mathrm{SiC}^{(11)}$.

We have applied the curving grid to the ab-initio calculation based on the Finite Element

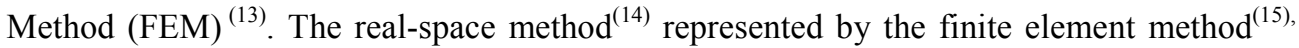
(16) has advantage for large-scale ab-initio calculation in point of suitability for parallel computation. Moreover, the FEM-based method yields a favorable convergence behavior of total energy. The error of total energy monotonically decreases in line with $\mathrm{O}\left(h^{2 n}\right)$ convergence rate with $n$-th order of $C^{0}$ shape function where $h$ is the size of a element. Once this convergence rate is known, we predict the element size required for a desired accuracy. In the density-functional norm-conserving pseudopotential calculation, this convergence rate is investigated by Pask et. $a l^{(15)}$ in the case of uniform mesh. The rate in the case of curving-grid mesh has been under investigation. The aim of this paper is to clarify how the convergence of the error behaves in the total-energy calculation with curving grid. We define the error using the reference energy obtained by an established plane-wave code, ABINIT $^{(17)}$. ABINIT allows us to use the same DFT functional with our FEM code. It is enable to compare the total energies directly. We show the effectiveness of curving-grid mesh in the FEM-based total energy calculation of oxide, $\mathrm{MgO}$.

The rest of this paper is organized as follows: in Section 2, we describe the FEM-based DFT scheme and the total-energy expression as a criterion of accuracy. The details of generating curving-grid mesh are also described in this section. The result of total energy calculation and a discussion on its convergence property are presented in Section 3. The last section contains our conclusions.

\section{Method}

\subsection{Kohn-Sham equation and total energy of DFT functional}

In this paper, we treat the restricted system where the electrons of up-spin and down-spin are admitted in the same orbital. In addition, we use the $\Gamma$-point sampling for the calculation so that the wave functions take real value. The Kohn-Sham equation as the governing equation of the $\mathrm{DFT}^{(1)}$ is described as

$$
\left(-\frac{1}{2} \nabla^{2}+v_{\mathrm{KS}}\right) \psi_{i}=\varepsilon_{i} \psi_{i} \quad i=1, \cdots, N_{\text {orbital }}
$$

where $\psi_{i}$ is the one-particle valence-electron wave function of the $i$-th orbital. $\varepsilon_{i}$ is the Kohn-Sham eigenenergy of $i$-th orbital. The number of wave functions is denoted by $N_{\text {orbital }}$. The Kohn-Sham effective potential $v_{\mathrm{KS}}$ based on the norm-conserving pseudopotential method $^{(10)}$ is given as

$$
v_{\mathrm{KS}}=\sum_{j}^{N_{\text {atom }}}\left(\hat{v}_{\mathrm{NL}, j}+v_{\mathrm{L}, j}\right)+\mu_{\mathrm{XC}}+v_{\mathrm{H}}
$$

where $\hat{v}_{\mathrm{NL}, j}$ is the nonlocal pseudopotential operator which represents the effect of core-electrons of $j$-th atom to a valence electron. The number of atoms is denoted by $N_{\text {atom }}$. The local pseudopotential $v_{\mathrm{L}, j}$ is the ionic core potential shielded by the core electrons. Under the local density approximation, we regard the exchange-correlation potential $\mu_{\mathrm{XC}}$ as a function of electron density $\rho$ defined as

$$
\rho=\sum_{i}^{N_{\text {orbital }}} f_{i} \psi_{i}^{*} \psi_{i}
$$

where $\psi_{i}^{*}$ is the complex conjugate of $\psi_{i}$. The partial occupancy $f_{i}$ follows the 
Fermi-Dirac-like distribution and determines the density $\rho$ which satisfies the following relationship:

$$
N_{\text {electron }}=\int \rho \mathrm{d} \Omega
$$

where $N_{\text {electron }}$ and $\Omega$ are electronic charge in the system and cell volume, respectively. The Hartree potential $v_{\mathrm{H}}$ which represents the Coulomb interaction between electrons is obtained by solving the Poisson equation.

$$
\nabla^{2} v_{\mathrm{H}}=-4 \pi \rho
$$

Solving the Kohn-Sham equation is a non-linear eigenvalue problem because the latter two potentials in Eq. (2) depend on wave functions thorough electron density $\rho$. The Kohn-Sham equation should be solved under a condition of orthonormality of wave functions given as

$$
\int \psi_{i}^{*} \psi_{j} \mathrm{~d} \Omega=\delta_{i j}
$$

By solving the non-linear problem under the condition of orthonormality, we can obtain a set of wave function, eigenvalues, electron density, potentials and partial occupancies as the ground state of DFT functional.

Using these variables which yield the ground state, the total energy $E_{\text {tot }}$ of the atomic system under periodic boundary condition is calculated as

$$
E_{\text {tot }}=\sum_{i}^{N_{\text {orbial }}} f_{i} \varepsilon_{i}-\frac{1}{2} \int v_{\mathrm{H}} \rho \mathrm{d} \Omega+\int\left(\varepsilon_{\mathrm{XC}}-\mu_{\mathrm{XC}}\right) \rho \mathrm{d} \Omega+\gamma_{\mathrm{EWALD}}-\sigma \sum_{i}^{N_{\text {orbial }}} S\left(f_{i}\right)
$$

where $\gamma_{\text {EWALD }}$ is a periodic interatomic potential given by Ewald scheme ${ }^{(18)}$. The last term in the right-hand side is the entropy term where $\sigma$ is the smearing parameter. This entropy term is introduced because we smear the occupancy $f_{i}$ in order to improve the conevergence speed of the total energy with respect to the number of k-points in metallic systems. As the smearing method, we employ the Gaussian smearing method in which the specific form of $S\left(f_{i}\right)$ is given in Ref. (19). Strictly speaking, the $E_{\text {tot }}$ in Eq. (7) is not referred as total energy but free energy because it includes the entropy term. However, we regard this energy expression as total energy in this paper. $\varepsilon_{\mathrm{XC}}$ is a exchange-correlation energy density which is a function of $\rho$ as well as $\mu_{\mathrm{XC}}$. The relationship between $\varepsilon_{\mathrm{XC}}$ and $\mu_{\mathrm{XC}}$ under the local density approximation ${ }^{(1)}$ is given as

$$
\mu_{\mathrm{XC}}=\varepsilon_{\mathrm{XC}}+\rho \frac{\partial \varepsilon_{\mathrm{XC}}}{\partial \rho}
$$

\subsection{FEM-discretized Kohn-Sham equation and total energy}

Using FEM shape function $\{N\}$, an arbitrary function $q(\mathbf{r})$ is interpolated as

$$
q(\mathbf{r})=\{N(\mathbf{r})\}^{T}\{q\}
$$

where vector $\{q\}$ is the nodal value of the function $q(\mathbf{r})$. We employ the FEM formulation based on the Galerkin weighted residual method ${ }^{(20)}$. The FEM-discretized Kohn-Sham equation is given as

$$
\left[\frac{1}{2}[L]+[V]+\sum_{j=1}^{N_{\text {atom }}} \sum_{l, m}\left\{p_{l, m, j}\right\}\left\{p_{l, m, j}\right\}^{\mathrm{T}}\right]\left\{\psi_{i}\right\}=\varepsilon_{i}[S]\left\{\psi_{i}\right\}
$$

where $\left\{\psi_{i}\right\}$ is the nodal wave function. The matrices in Eq. (7) are given as follows:

$$
[L]=\sum_{\mathrm{e}} \int_{\Omega_{\mathrm{e}}}[B]^{\mathrm{T}}[B] \mathrm{d} \Omega_{\mathrm{e}}
$$




$$
\begin{gathered}
{[V]=\sum_{\mathrm{e}} \int_{\Omega_{\mathrm{e}}}\left(\sum_{j=1}^{N_{\text {atom }}} v_{\mathrm{L}, j}+\mu_{\mathrm{XC}}+v_{\mathrm{H}}\right)\{N\}\{N\}^{\mathrm{T}} \mathrm{d} \Omega_{\mathrm{e}}} \\
{[S]=\sum_{\mathrm{e}} \int_{\Omega_{\mathrm{e}}}\{N\}\{N\}^{\mathrm{T}} \mathrm{d} \Omega_{\mathrm{e}}}
\end{gathered}
$$

$[B]$ is constituted by the shape function vector and the matrix of spatial derivatives of the shape function. $\Omega_{\mathrm{e}}$ is a occupied region by a finite element. $\Sigma_{\mathrm{e}}$ represents assembling all the elements. The vector $\left\{p_{l, m, j}\right\}$ in Eq. (8) is the component of the Kleinman-Bylander nonlocal pseudopotential $^{(21)}$ given as

$$
\left\{p_{l, m, j}\right\}=\sum_{\mathrm{e}} \int p_{l, m, j}(\mathbf{r})\{N\} \mathrm{d} \Omega_{\mathrm{e}} .
$$

The pseudopotential $p_{l, m, j}$ is a function on the coordinate. We employ the GTH pseudopotential and the specific form of the function $p_{l, m, j}$ is described in Ref. (22). The specific form of $\mu_{\mathrm{XC}}$ and $v_{\mathrm{L}, j}$ are also given in Ref. (22). The integrations in Eq. (9) - (12) are performed numerically where all the values in integrand are estimated on integral points and summed in line with the Gauss quadrature ${ }^{(23)}$. We should choose the appropriate numbers of integral point in each integrand of Eq. (9) - (12) so as to achieve enough accuracy of the total energy. In potential calculation on the integral point, divergence term in the Hartree potential and local potential due to periodic boundary condition need to be removed. The Ewald scheme ${ }^{(18)}$ is used to remove the divergence term from the local pseudopotential, specific form of which is described in Ref. (22). The Hartree potential is calculated by solving the following FEM-discretized Poisson equation,

$$
[L]\left\{v_{\mathrm{H}}\right\}=4 \pi[S]\left\{\rho^{\prime}\right\}
$$

where $\left\{v_{\mathrm{H}}\right\}$ is the nodal value of the Hartree potential. The vector $\left\{\rho^{\prime}\right\}$ is the nodal value of $\rho^{\prime}$ calculated by substituting the level of electron density $N_{\text {electron }} / \Omega$ from the density $\rho$. The Hartree potential on the integral point is obtained by interpolation from the nodal value $\left\{v_{\mathrm{H}}\right\}$. The FEM-discretized Kohn-Sham equation is solved by the non-linear solver such as conjugate gradient method.

The total energy in Eq. (7) is rewritten in the following expression:

$$
E_{\mathrm{tot}}=\sum_{i}^{N_{\text {otitial }}} f_{i} \varepsilon_{i}+\sum_{\mathrm{e}} \int_{\Omega_{\mathrm{e}}}\left(-\frac{1}{2} v_{\mathrm{H}}+\varepsilon_{\mathrm{XC}}-\mu_{\mathrm{XC}}\right)\{N\}^{\mathrm{T}} \mathrm{d} \Omega_{\mathrm{e}}\{\rho\}+\gamma_{\mathrm{EWALD}}-\sigma \sum_{i}^{N_{\text {ortial }}} S\left(f_{i}\right)
$$

In this paper, we employ this total-energy expression for a criterion to estimate the accuracy of calculation.

\subsection{Curving grid}

Even in the norm-conserving pseudopotential technique, the distribution of wave function which represents the behavior of valence electron shows large variation around the nuclei. The variation is large especially around the nucleus of first-row or $3 \mathrm{~d}$ transition atoms, which have strongly localized $p$ or $d$ valence orbitals because these atoms have no $p$ or $d$ core orbitals near the valence orbitals. As an example, the distribution of local pseudopotential of $\mathrm{O}$ atom and $\mathrm{Mg}$ atom are shown in Fig.1. The local pseudopotential of $\mathrm{O}$ atom is much deeper than that of $\mathrm{Mg}$ atom around the nucleus. A fine mesh is required around the nucleus to achieve the accurate total-energy calculation since the large variation becomes the main source of the error in the total energy. In order to enhance the performance of the finite element calculation, using a nonuniform mesh which have fine mesh around the nuclei is effective. As a non-uniform mesh, we use a curving-grid mesh generated by the deformation-function method $^{(11)}$, which gives a mapping from an curvilinear coordinate $\zeta$ to an Euclidean coordinate $\mathbf{x}$ using the deformation function $g_{j}$ as 


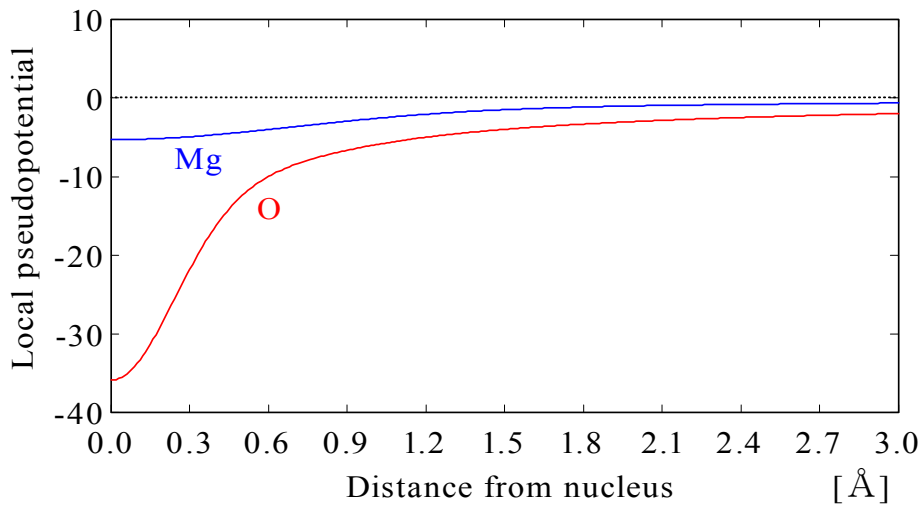

Fig.1. Local pseudopotential of $\mathrm{O}$ atom and $\mathrm{Mg}$ atom

$$
\zeta(\mathbf{x})=\mathbf{x}+\sum_{j}^{N \text { atoms }} \sum_{\mathbf{P}}\left(\mathbf{x}-\mathbf{u}_{j}-\mathbf{P}\right) g_{j}\left(\left|\mathbf{x}-\mathbf{u}_{j}-\mathbf{P}\right|\right)
$$

where $\mathbf{u}_{j}$ and $\mathbf{P}$ are the position of $j$-th atom and the super-cell vector, respectively. We use the deformation function $g_{j}$ proposed by $\mathrm{Gygi}^{(24)}$,

$$
g_{j}(r)=A_{j} \operatorname{sech} \frac{r}{a_{j}}
$$

where $A_{j}$ and $a_{j}$ are the parameters of $j$-th atom which are chosen so as to give the appropriate result in total energy calculation. In the original studies of deformation-function method, the calculation is performed in a mapping space of uniform mesh because the basis function needs to be defined in uniform mesh. However, our FEM shape function as a basis function can be defined in nonuniform mesh. Thus we generate the curving-grid mesh using the deformation-function method and the calculation is performed on the nonuniform mesh directly. In generation of the curving-grid, the non-linear equation of Eq. (17) is solved by the Newton-Raphson method. An example of a mapping from the Euclidean coordinate to the curvilinear coordinate for $\mathrm{O}_{2}$ molecule calculation is shown in Fig. 2.

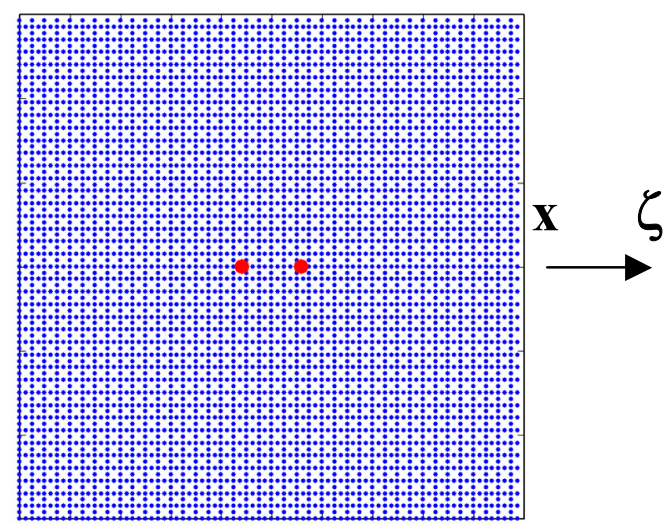

Euclidean coordinate $\mathbf{x}$

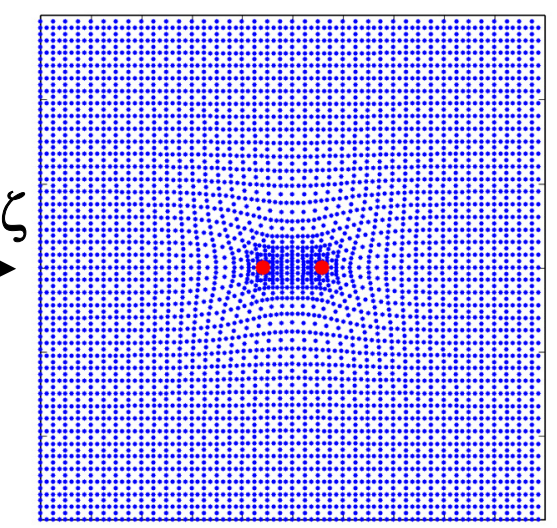

Curvilinear coordinate $\zeta$

Fig.2. Positions of FEM node on intersection $\left(\begin{array}{lll}0 & 0 & 2\end{array}\right)$ for $\mathrm{O}_{2}$ molecule: curvilinear coordinate $\zeta$ and Euclidean coordinate $\mathbf{x}$

The positions of the nodes on the intersection $\left(\begin{array}{lll}0 & 0 & 2\end{array}\right)$ in Miller indices are shown in this figure. Both parameters of $A_{j}$ and $a_{j}$ are set to 1.0. In the curvilinear coordinate, the mesh around the oxygen nucleus is finer than that of the Euclidean coordinate, in contrast, the mesh far from the nucleus is coarser. Such a mesh redistribution technique is called r-type refinement ${ }^{(25)}$, which seems less efficient compared to the other mesh refinement 
techniques, e.g. h-type or p-type ${ }^{(25)}$, because the r-type refinement only redistributes the mesh. However, we employ this method because it provides the easiest way to generate a non-uniform grid which has the fine resolution around the nuclei.

\section{Computational result and discussion}

\subsection{Computational details}

We use a periodic super-cell as the computational region and only $\Gamma$ point is used for the k-point sampling ${ }^{(9)}$. The initial wave functions and electron density are constructed from linear superposition of the atomic orbitals and densities calculated by Gaussian98 ${ }^{(26)}$, respectively. The Gaussian smearing method ${ }^{(27)}$ is used to introduce the partial occupancy. The Gaussian smearing parameter is set to 0.02. In the FEM discretization, the wave functions, electron density and Hartree potential are represented by the linear combinations of the FEM shape functions. As the shape function, we employ the second-order serendipity FEM shape function ${ }^{(28)}$. The shape of each finite element is hexahedron. By using the domain-decomposition technique ${ }^{(29)}$, the following calculations are parallelized on the 8 -node PC cluster. The Blocked-Jacobi-ICC(0)-CG linear solver is employed to solve the Poisson equation $^{(30)}$. The linearized Kohn-Sham equation is solved by a band-by-band iterative solver, i.e. the BKL-CG ${ }^{(31)}$ with sub-space-rotation method ${ }^{(19)}$.

In error estimation of the FEM calculation, $\mathrm{ABINIT}^{(17)}$ is used to obtain the reference energy. ABINIT is well-developed software on the basis of the plane wave method. In this paper, the error of calculation $e$ is defined as the deviation of energies given as

$$
e=\left(E_{\mathrm{FEM}}-E_{\mathrm{ABINIT}}\right) / N_{\text {atom }}
$$

where $E_{\mathrm{FEM}}$ is the free energy obtained by Eq. (16) in our FEM calculation, $E_{\mathrm{ABINIT}}$ is the reference energy obtained by ABINIT. The energies obtained by both code can be directly compared because the pseudopotential and the LDA functional are common.

\subsection{Calculation of bulk hcp Mg on uniform mesh}

We estimate the error in the calculation of bulk hcp Mg including 64 atoms using uniform mesh. The crystal structure of the bulk hep $\mathrm{Mg}$ is shown in Fig. 3. This calculation is performed using the uniform mesh and the size of periodic cell is $12.84 \times 11.12 \times 10.42 \AA^{3}$. The division of the cell is adjusted so as to give the nearly cubic finite element. The convergence behavior of error as a function of element size is shown in Fig. 4.

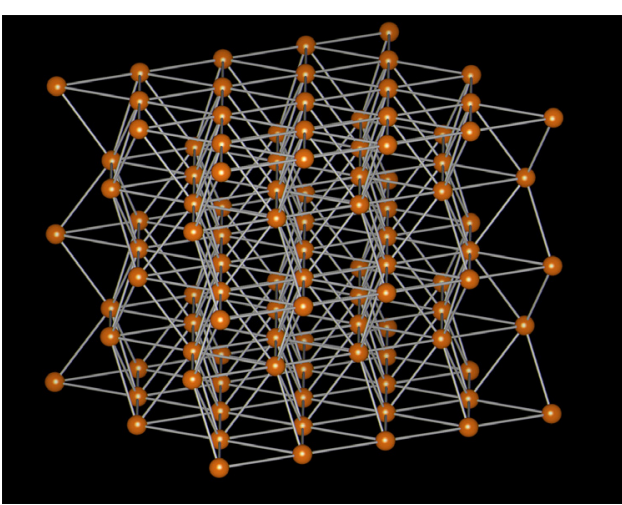

Fig. 3. Crystal structure of bulk hep $\mathrm{Mg}$

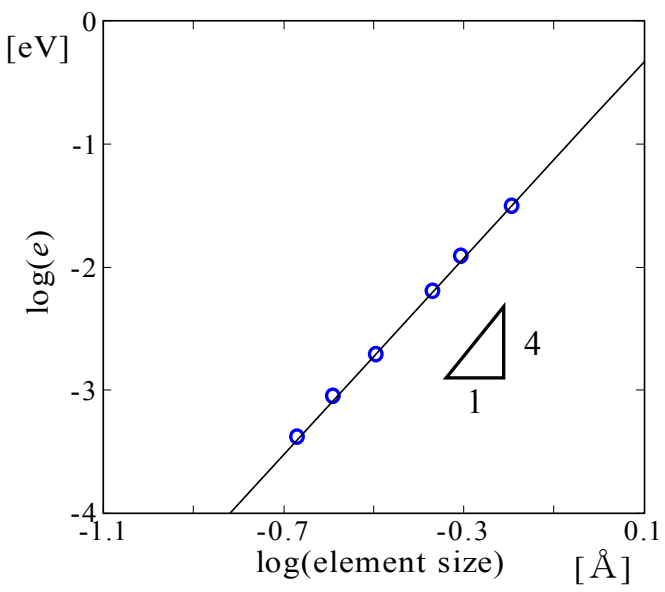

Fig. 4. Convergence behavior of error in calculation of bulk hcp Mg 
The element size in this figure indicates the longest edge of a finite element. The reference energy is obtained by ABINIT with a cut-off energy, $1632 \mathrm{eV}$. The change of the total energy is less than $1.0 \times 10^{-5} \mathrm{eV}$ when the cut-off energy increases from $1360 \mathrm{eV}$ to 1632 $\mathrm{eV}$. The logarithm of error as a function of the logarithm of the element size decreases monotonically with the element-size reduction and has the gradient of about 4.0. It means that our FEM formulation yields variational convergence of the error and gives an order of error $O\left(\right.$ element $\left.\operatorname{size}^{4}\right)$. This order of error is consistent with the error analysis performed in the FEM formulation for a one-dimensional Schrödinger equation with the second-order Lagrange shape function ${ }^{(32)}$.

\subsection{Calculation of $\mathrm{O}_{2}$ molecule on uniform and curving grid}

We compare the convergence behaviors of the error in calculation of $\mathrm{O}_{2}$ molecule using uniform mesh and curving-grid mesh. The periodic cell is set by cube of edge length 12.00 $\AA$. The bond length of $\mathrm{O}_{2}$ molecule is set to $1.2 \AA$. The reference energy is obtained by ABINIT with a cut-off energy, $3810 \mathrm{eV}$. The change of the total energy is less than $2.0 \times 10^{-2} \mathrm{eV}$ when the cut-off energy increases from $3265 \mathrm{eV}$ to $3810 \mathrm{eV}$. The curving-grid mesh shown in Fig. 2 is employed. The result is shown in Fig. 5. The error levels calculated by ABINIT with cut-off energy $1904 \mathrm{eV}$ are also shown in this figure. The element size of curving grid corresponds to the element size of the uniform mesh from which the curving-grid mesh generates. As well as the result of bulk hcp $\mathrm{Mg}$, the convergence of error in uniform mesh is variational and the order of error is nearly $O\left(\right.$ element $\left.\operatorname{size}^{4}\right)$. We have found that this convergence property is also conserved even in the calculation with curving-grid mesh. By using curving grid the convergence line is shifted to the right with identical gradient. This is important because this convergence property yields rough estimation of the element size required for a desired accuracy. The element size required for the accuracy corresponding to $1904 \mathrm{eV}$ cut-off energy is predicted as about $0.11 \AA$ in the case of uniform mesh. In the case of curving-grid mesh the element size required is about $0.22 \AA$, which is double of element size required in the case of uniform mesh. This means that the number of degrees of freedom can be reduced to $(1 / 2)^{3}$ by using the curving-grid mesh.

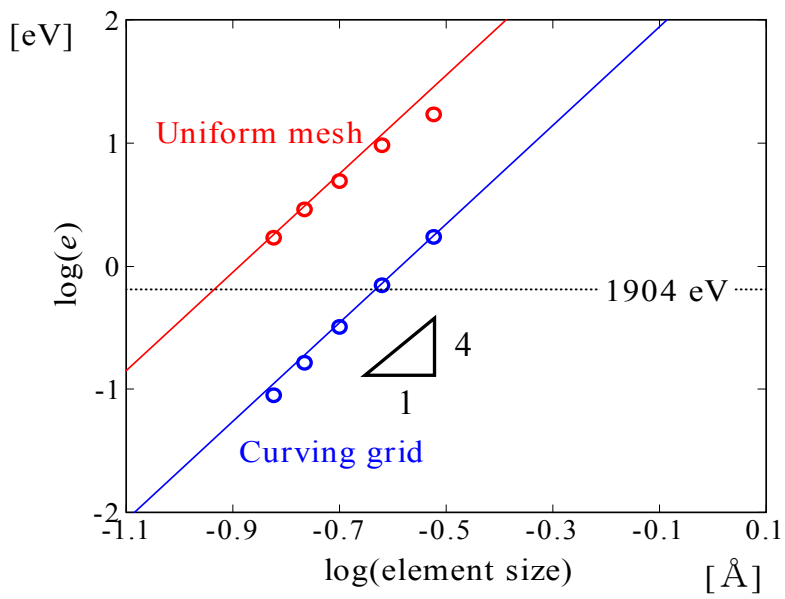

Fig. 5. Convergence behaviors of error in calculation of $\mathrm{O}_{2}$ molecule using uniform mesh and curving grid mesh

\subsection{Calculation of MgO ceramic on curving grid}

We apply our FEM program with curving-grid mesh to a calculation of bulk rock-salt $\mathrm{MgO}$ ceramic including 216 atoms. The periodic cell is set by cube of edge length $12.64 \AA$. 
The crystal structure is shown in Fig. 6 where small spheres indicate the positions of $\mathrm{O}$ atom. We set the curvilinear parameter in Eq. (17) only on $\mathrm{O}$ atom because the local pseudopotential of $\mathrm{O}$ atom around the nucleus is much deeper than that of $\mathrm{Mg}$ as shown in Fig. 1. The positions of FEM node on the intersection $\left(\begin{array}{lll}0 & 0 & 2\end{array}\right)$ in Miller indices are shown in Fig.

7. The fine mesh is generated around the nuclei of $\mathrm{O}$ atom. The density distribution of the valence electron on the intersection $\left(\begin{array}{lll}0 & 0 & 2\end{array}\right)$ is shown in Fig. 8. Contours in the

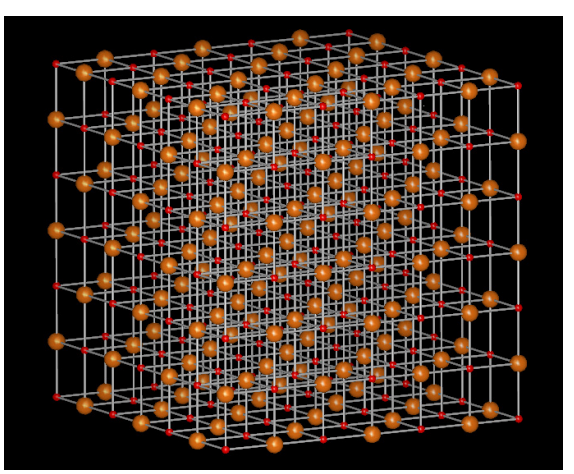

Fig. 6. Crystal structure of bulk rock-salt $\mathrm{MgO}$ including 216 atoms $\left(\begin{array}{lll}0 & 0 & 2\end{array}\right)$ plane are plotted from 0.007 electron $/ \AA^{3}$ to 7.259 electron $/ \AA^{3}$ in spacing of 0.427 electron $/ \AA^{3}$. The red lines correspond to the contours line of 0.007 electron $/ \AA^{3}$. The valence electrons are delocalized around the $\mathrm{Mg}$ nuclei and are highly localized around the $\mathrm{O}$ nuclei. In such a ionic-bonding ceramic system, curving-grid mesh is especially effective for the reduction of the number of freedom. In Table 1, the total energy obtained by the FEM with uniform mesh and the curving-grid mesh are compared with the result by ABINIT with the cut-off energy 1088 $\mathrm{eV}$, which is enough accurate to predict most of material properties of $\mathrm{MgO}^{(33),(34)}$. In both FEM calculations, the element size is set to $0.30 \AA$ which is the largest size in the cases of $\mathrm{O}_{2}$ calculation in Fig. 5. The accuracy of the calculations is proven by the less total energy since the FEM and ABINIT are formulated on the basis of the variational principle and the same DFT functional. As shown in Table 1, FEM with curving-grid mesh yields more accurate total energy than by ABINIT while the accuracy of the result with uniform mesh is inferior by $1000 \mathrm{eV}$ to that of the result by ABINIT. Using curving-grid mesh we can obtain enough achievement for the prediction of material properties of $\mathrm{MgO}$.

Table 1 Total energy of bulk rock-salt MgO including 216 atoms

\begin{tabular}{l|c}
\multicolumn{1}{c|}{ method } & total energy $[\mathrm{eV}]$ \\
\hline ABINIT with cut-off energy, $1088 \mathrm{eV}$ & -49660.87 \\
\hline FEM with uniform mesh & -48490.56 \\
\hline FEM with curving grid & -49845.79
\end{tabular}

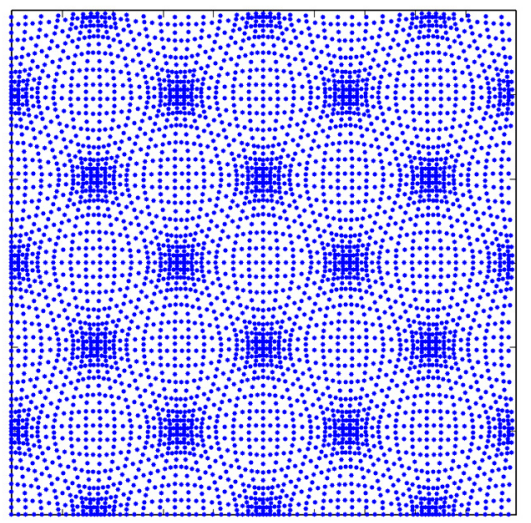

Fig. 7. Positions of FEM node on intersection (llll $\left.\begin{array}{lll}0 & 0 & 2\end{array}\right)$ for bulk rock-salt $\mathrm{MgO}$ including 216 atoms

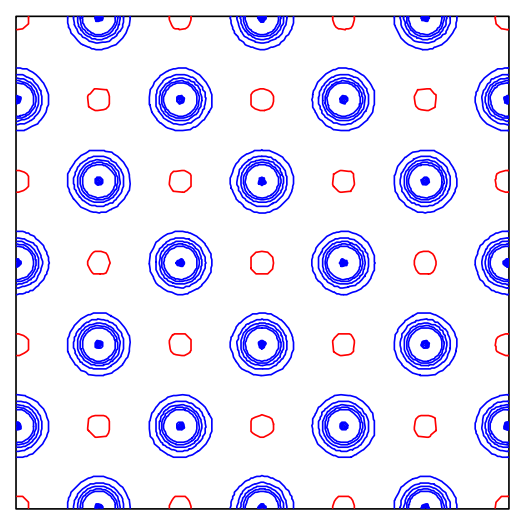

Fig. 8. Density distribution of valence electrons on intersection $\left(\begin{array}{lll}0 & 0 & 2\end{array}\right)$ for bulk rock-salt $\mathrm{MgO}$ including 216 atoms 


\section{Conclusions}

In this paper, we have shown the applicability of curving-grid mesh in the ab-initio finite-element calculation. We investigate the convergence property of the total-energy calculation with curving-grid mesh in the system including $\mathrm{O}$ atoms, which has the highly localized wave function around the nuclei. The favorable convergence property, i.e. the variational convergence with the uniform rate, of the finite element method with uniform mesh is conserved. This allows us to estimate a rough size of the element required for a desired accuracy. The calculation of bulk rock-salt $\mathrm{MgO}$ ceramic including 216 atoms is demonstrated by using curving-grid mesh. The accuracy of the calculation is higher than the result obtained by ABINIT with a cut-off energy, $1088 \mathrm{eV}$, which is sufficient for the prediction of most of material properties of $\mathrm{MgO}$. This result proves the usability of ab-initio finite-element calculation with curving-grid mesh in the prediction of material properties with high accuracy.

\section{Acknowledgement}

This work was supported by the Grant-in-Aid (19560073) for Scientific Research by the Ministry of Education, Science, and Culture of Japan.

\section{References}

(1) Kohn W. and Sham L. J., Self-consistent equations including exchange and correlation effects, Physical Review, Vol. 140, (1965), pp. A1133-A1188.

(2) Reimann S. M. and Manninen M., Electronic structure of quantum dots, Reviews of Modern Physics, Vol. 74, (2002), pp. 1283-1342.

(3) Greeley J. and Nørskov J. K., Large-scale, density functional theory-based screening of alloys for hydrogen evolution, Surface Science, Vol. 601, (2007), pp. 1590-1598.

(4) Shi Z., Zhang J., Liu Z., Wang H. and Wilkinson D. P., Current status of ab initio chemistry study for oxygen electroreduction on fuel cell catalysis, Electrochimica Acta, Vol. 51, (2006), pp. 1905-1916.

(5) Hebbache M., First-principles calculations of the bulk modulus of diamond, Solid State Communication, Vol. 110, (1999), pp. 559-564.

(6) Clerc D. G. and Ledbetter H., Second-order and third-order elastic properties of diamond: An $a b$ initio study, Journal of Physics and Chemistry of Solid, Vol. 66, (2005), pp. 1589-1597.

(7) Li W. and Wang T., Elasticity, stability, and ideal strength of $\beta$-SiC in plane-wave-based $a b$ initio calculations, Physical Review, B, Vol. 6, 59 (1999), pp. 3993-4001.

(8) Yoo M. H., Zou J. and Fu C. L., Mechanistic modeling of deformation and fracture behavior in $\mathrm{TiAl}_{3}$ and $\mathrm{Ti}_{3} \mathrm{Al}$, Materials Science and Engineering, A, Vol. 192/193, (1995), pp. 14-23.

(9) Payne M. C., Teter M. P., Allan D. C., Arias T. A. and Joannopoulos J. D., Iterative minimization techniques for ab-initio total-energy calculations: molecular dynamics and conjugate gradient, Reviews of Modern Physics, Vol. 64, (1992), pp. 1045-1097.

(10) Bachelet G. B., Hamann D. R., and Schlüter M., Pseudopotentials that work: From H to Pu, Physical Review, B, Vol. 26, (1982), pp. 4192-4228.

(11) Hamann D. R., Comparison of global and local adaptive coordinate for density-functional calculations, Physical Review, B, Vol. 63, (2001), pp. 075107-1-075107-7.

(12) Hamann D. R., Adaptive-coordinate electronic structure of $3 d$ bands: $\mathrm{TiO}_{2}$, Physical Review, B, Vol. 56, (1997), pp. 14979-14984.

(13) Shiihara Y., Band-by-band ab-initio finite element method for large metallic systems, $P h . D$. Thesis, Univ. of Tokyo, (2007).

(14) Beck T. L., Real-space mesh techniques in density-functional theory, Reviews of Modern Physics, Vol. 72, (2000), 1041-1080. 
(15) Pask J. E. and Sterne P. A., Finite element methods in ab initio electronic structure calculations, Modelling and Simulation in Material Science and Engineering, Vol. 13, (2005), pp. R71-R96.

(16) Tsuchida E. and Tsukada M., Large-Scale Electronic-Structure Calculations Based on the Adaptive Finite-Element Method, Journal of Physics Society of Japan, Vol. 67, (1998), pp. 3844-3855.

(17) Gonze X., Beuken J.-M., Caracas R., Detraux F., Fuchs M., Rignanese G.-M., Sindic L., Verstraete M., Zerah G., Jollet F., Torrent M., Roy A., Mikami M., Ghosez Ph., Raty J.-Y. and Allan D.C., First-principles computation of material properties : the ABINIT software project, Computational Materials Science, Vol. 25, (2002), pp. 478-492.

(18) Ewald P.P., Die Berechnung optischer und elektrostatischer Gitterpotentiale, Annalen der Physik, Vol. 369, (1921), pp. 253-287.

(19) Kresse G. and Furthmuller J., Efficiency of ab-initio total energy calculations for metals and semiconductors using a plane-wave basis set, Computational Materials Science, Vol. 6, (1996), pp. 15-50.

(20) Zienkiewicz O. C. and Taylor R. L., The Finite Element Method, Vol. 2, (2000), p. 39, McGraw-Hill.

(21) Kleinmann L. and Bylander D. M., Efficacious Form for Model Pseudopotentials, Physical Review Letters, Vol. 48, (1982), pp. 1425-1428.

(22) Goedecker S., Teter M. and Hutter J., Separable dual-space Gaussian pseudopotentials, Physical Review, B, Vol. 54, 3 (1996), pp. 1703-1709.

(23) Zienkiewicz O. C. and Taylor R. L., The Finite Element Method, Vol. 2, (2000), p. 219, McGraw-Hill.

(24) Gygi F., Ab initio molecular dynamics in adaptive coordinates, Physical Review, B, Vol. 51, 16 (1995), pp. 11190-11193.

(25) Zienkiewicz O. C. and Taylor R. L., The Finite Element Method, Vol. 2, (2000), p. 402, McGraw-Hill.

(26) Frisch M. J. et al., Gaussian 98 Revision A.11.4, (2002), Pittsburgh.

(27) $\mathrm{Fu} \mathrm{C}$. -L. and Ho K. -M., First-principles calculation of the equilibrium ground-state properties of transition metals: Applications to $\mathrm{Nb}$ and Mo, Physical Review, B, Vol. 28, (1983), pp. 5480-5486.

(28) Zienkiewicz O. C. and Taylor R. L., The Finite Element Method, Vol. 2, (2000), p. 174, McGraw-Hill.

(29) Smith B., Bjørstad P. and Gropp W., Domain Decomposition, Parallel Multilevel Methods for Elliptic Partial Differential Equations, (1996), p. 19, Cambridge University Press.

(30) Saad Y., Iterative Methods for sparse linear systems, (1996), p. 374, PWS.

(31) Bylander D. M., Kleinman L. and Lee S., Self-consistent calculations of the energy bands and bonding properties of $\mathrm{B}_{12} \mathrm{C}_{3}$, Physical Review, B, Vol. 42, 2 (1990), pp. 1394-1403.

(32) Ram-Mohan L. R., Finite Element and Boundary Element Applications in Quantum Mechanics, (2002), p. 84, Oxford.

(33) Shpakov V., Gotte A., Baudin M.,Woo T. and Hermansson K., MgO (001) surface phonons from ab initio calculations, Physical Review, B, Vol. 72, (2005), pp. 195427-1-195427-6.

(34) Organov A. R., Gillan M. J., Price G. D., Ab initio lattice dynamics and structural stability of MgO, Journal of Chemical Physics, Vol. 118, 22 (2003), pp. 10174-10182. 